\title{
Effect of virtual-assisted lung mapping in acquisition of surgical margins in sublobar lung resection
}

\author{
Masaaki Sato, MD, PhD, ${ }^{\text {a }}$ Masashi Kobayashi, $\mathrm{MD}, \mathrm{PhD},{ }^{\mathrm{b}}$ Fumitsugu Kojima, $\mathrm{MD}, \mathrm{PhD}$, \\ Fumihiro Tanaka, MD, PhD, ${ }^{\mathrm{d}}$ Masahiro Yanagiya, $\mathrm{MD},{ }^{\mathrm{e}}$ Shinji Kosaka, MD, PhD, ${ }^{\mathrm{f}}$ \\ Ryuta Fukai, MD, PhD, ${ }^{\mathrm{g}}$ and Jun Nakajima, $\mathrm{MD}, \mathrm{PhD}^{\mathrm{a}}$
}

\section{ABSTRACT}

Objective: Virtual-assisted lung mapping is a preoperative bronchoscopic multi-spot dye-marking technique. This study aimed to examine the efficacy of virtual-assisted lung mapping for obtaining sufficient surgical margins in sublobar lung resection.

Methods: The multicenter, prospective, single-arm study was conducted from September 2016 to July 2017 in 19 registered centers. Patients who required sublobar lung resection and careful determination of resection margins underwent virtual-assisted lung mapping followed by thoracoscopic surgery. Successful resection was defined as resection of the lesion with margins greater than the lesion diameter or $2 \mathrm{~cm}$ using the preoperatively planned resection without additional resection. We defined the primary goal of the study as achieving successful resection in $95 \%$ of lesions.

Results: The resection of 203 lesions (average diameter, $9.6 \pm 5.3 \mathrm{~mm}$ ) was intended in 153 patients. The lesions included pure and mixed ground-glass nodules (75 [35.9\%] and 36 [17.2\%], respectively), solid nodules (91 [43.5\%]), and others $(7[3.3 \%])$. Surgical procedures included wedge resection (131, $71.2 \%)$, segmentectomy $(51,27.7 \%)$, and others $(2,1.1 \%)$. Successful resection was achieved in 178 lesions (87.8\% [95\% confidence interval, 82.4-91.9\%]), and virtual-assisted lung mapping markings successfully aided in the identification of 190 lesions (93.6\% [95\% confidence interval, 89.3-96.5\%]). Multivariable analysis showed that the most significant factor affecting resection success was the depth of the necessary resection margin $(P=.0072)$.

Conclusions: This study showed that virtual-assisted lung mapping has reasonable efficacy, although the successful resection rate did not reach the primary goal. The depth of the required margin was the most significant factor leading to resection failure. (J Thorac Cardiovasc Surg 2018;156:1691-701)

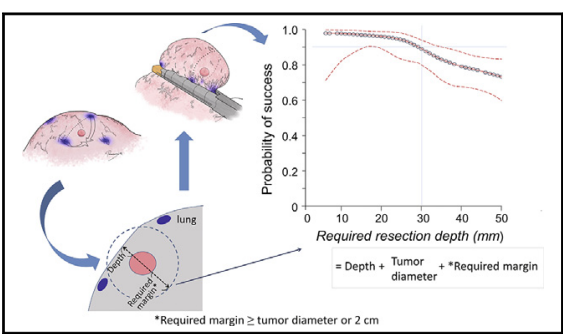

VAL-MAP has increased risk of insufficient margins when deeper resection lines are needed.

Central Message

VAL-MAP has reasonable efficacy, although the depth of the required margin was the most significant factor leading to resection failure in sublobar lung resection using the current VAL-MAP technique

\section{Perspective}

This multicenter prospective trial in Japan revealed that VAL-MAP, a bronchoscopic multi-spot dye marking in the lung, enables effective tumor localization and determination of resection lines in most sublobar lung resection cases. However, in the current VAL-MAP technique, the risk of insufficient margins increases as the required depth of the resection line increases.

See Editorial Commentary page 1702.
Small pulmonary nodules with or without the radiologic characteristics of ground-glass opacity (GGO) are frequently encountered in clinical practice. Although high-resolution computed tomography (CT) depicts these

\footnotetext{
From the a Department of Thoracic Surgery, The University of Tokyo, Tokyo, Japan; ${ }^{\mathrm{b}}$ Department of Thoracic Surgery, Tokyo Medical and Dental University, Tokyo, Japan; 'Department of Thoracic Surgery, St Luke's International Hospital, Tokyo, Japan; ${ }^{\mathrm{d}}$ The Second Department of Surgery, University of Occupational and Environmental Health, Kitakyushyu, Japan; ${ }^{\mathrm{e}}$ Department of Thoracic Surgery, NTT Medical Center Tokyo, Tokyo, Japan; ${ }^{\mathrm{f}}$ Department of Thoracic Surgery, Shimane Prefectural Central Hospital, Izumo, Japan; and ${ }^{\mathrm{g}}$ Department of Thoracic Surgery, Shonan Kamakura General Hospital, Kamakura, Japan.

The study was funded by the Japan Agency for Medical Research and Development. The clinical trial registry number is UMIN000022991. This study was approved by the Ethics Committee at the University of Tokyo on February 29, 2016 (P201503711 ) and by the ethics committees of each participating institution. The entire study was approved by the Advanced Medical Technology Review Board (June 16, 2016)
}

lesions clearly, it is sometimes challenging for surgeons to localize them intraoperatively. Sublobar lung resection procedures such as wedge resection and segmentectomy can be performed for such small nodules, although the

and Advanced Medical Treatment Committee (August 4, 2016) of the Japanese Ministry of Health, Labour, and Welfare.

Read at the 98th Annual Meeting of The American Association for Thoracic Surgery, San Diego, California, April 28-May 1, 2018.

Received for publication Jan 15, 2018; revisions received April 25, 2018; accepted for publication May 11, 2018.

Address for reprints: Masaaki Sato, MD, PhD, Department of Thoracic Surgery, The University of Tokyo Hospital, 7-3-1 Hongo, Bunkyo-ku, Tokyo 113-8655, Japan (E-mail: satom-sur@h.u-tokyo.ac.jp).

0022-5223

Copyright (C) 2018 by The American Association for Thoracic Surgery. Published by Elsevier Inc. This is an open access article under the CC BY-NC-ND license (http:// creativecommons.org/licenses/by-nc-nd/4.0/).

https://doi.org/10.1016/j.jtcvs.2018.05.122 


\section{Abbreviations and Acronyms}

$\mathrm{CI}=$ confidence interval

$\mathrm{CT} \quad=$ computed tomography

GGO = ground-glass opacity

VAL-MAP $=$ virtual-assisted lung mapping

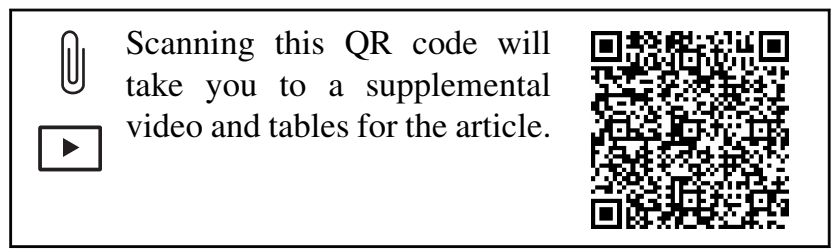

indication and required safe margin for lung cancer and metastatic lung tumors remain controversial. ${ }^{1,2}$

Virtual-assisted lung mapping (VAL-MAP) is a relatively novel, bronchoscopic, preoperative, multi-spot dye marking technique that uses virtual images (eg, virtual bronchoscopy) to assist in identifying lesions intraoperatively (Video 1). ${ }^{3}$ VAL-MAP was originally developed in Japan to overcome the limitations of CT-guided percutaneous needle-mediated marking techniques, especially the hookwire method, wherein potentially fatal air embolism was reported in $1 \%$ to $2 \%$ of cases. ${ }^{4-8}$ VAL-MAP is reportedly safe, with no reported cases of air embolism and limited complications even after the accumulation of 500 cases in a multicenter trial. ${ }^{9}$ In addition to localization of small nodules, the multiple marks function as reference points of the "lung map" on the lung surface. The lung map assists surgeons to determine appropriate resection lines in wedge resections and segmentectomies, especially in extended or complex segmentectomies, ${ }^{10-12}$ although it is not mandatory to place marks on resection lines. However, a previous multicenter prospective study in Japan showing a successful resection rate of $99 \%$ did not take surgical margins into consideration. ${ }^{10}$

Acquisition of resection margins is critical in limited lung resections. Insufficient resection margins in limited resections are reportedly associated with a greater incidence of local regional recurrence ${ }^{13-16}$ and are potentially associated with poorer clinical outcomes. ${ }^{17}$ Beyond the concept of tumor localization in conventional marking techniques, VAL-MAP has the potential to design limited lung resection surgery that guarantees sufficient resection margins.

We hypothesized that VAL-MAP would enable efficient determination of satisfactory resection lines. The primary purpose of the present study was to rigorously examine the impact of VAL-MAP on resection margins in thoracoscopic lung resection.

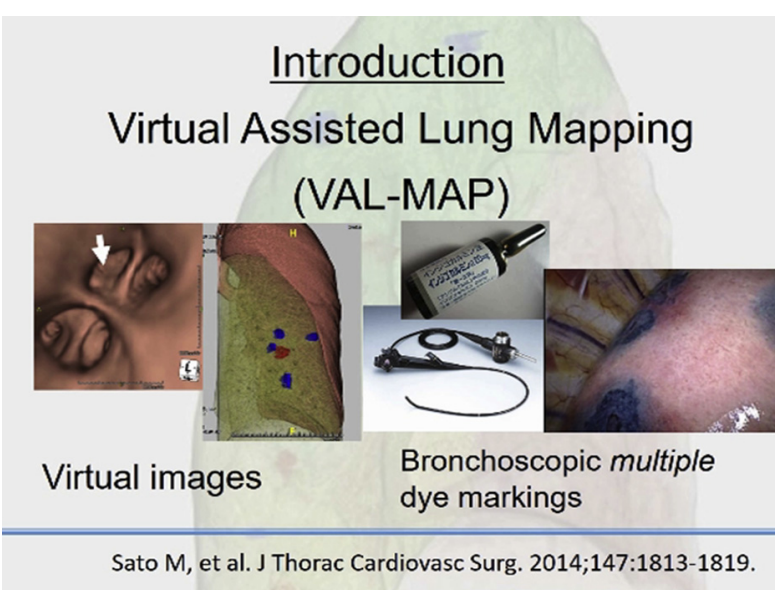

VIDEO 1. The video shows the background, methods including the technique of VAL-MAP, and results of the present study. Video available at: https://www.jtcvs.org/article/S0022-5223(18)31832-4/fulltext.

\section{MATERIALS AND METHODS}

A multicenter, prospective, single-arm study was conducted from September 2016 to July 2017 under the supervision of the Japanese Ministry of Health, Labour, and Welfare to evaluate the safety and efficacy of VAL-MAP. The study protocol is shown in the Online Data Supplement.

\section{Patient Registration}

Patients meeting the inclusion and exclusion criteria described in Table E1 were enrolled after providing informed consent. Briefly, patients with pulmonary nodule or nodules that showed the following characteristics were enrolled: suspected or diagnosed as malignant, requiring sublobar lung resection (ie, wedge resection or segmentectomy) for curative treatment, and anticipated to require careful determination of resection lines to ensure resection margins because of difficult intraoperative tumor localization (eg, due to a GGO component, diameter $\leq 5 \mathrm{~mm}$, or diameter equal to or less than the distance from the visceral pleura). A present or past medical history of bronchial asthma was considered a relative contraindication, and if the risk was assessed as greater than the potential benefit to the patient, he or she was excluded from the study as per exclusion criterion III. Otherwise, a bronchodilator was used prophylactically following the bronchoscopic guidelines issued by the safety committee of the Japan Society for Respiratory Endoscopy ${ }^{18}$ and the guidelines issued by the British Thoracic Society. ${ }^{19}$

\section{Mapping Procedure}

The mapping procedure was conducted on the day of surgery, or 1 or 2 days preoperatively, on the basis of the data obtained in a previous clinical trial. ${ }^{10}$ The multiple spots on the lung surface or lung map were designed on the basis of CT images and corresponding virtual bronchoscopy, and the target bronchi were identified. The workstation used for this process was not specified, but was dependent on the preferences of each center. The details of the bronchoscopic marking technique have been described. ${ }^{3,8,9}$ In short, under sedation and local anesthesia, thoracic surgeons or respirologists conducted the bronchoscopic procedure while monitoring blood pressure, electrocardiography, and oxygenation. The bronchoscope (BF-260F or BF-P260F; Olympus, Tokyo, Japan) was orally inserted. Once the target bronchi were identified using prepared virtual bronchoscopy as guidance, a metal blunt-tip catheter (P6-CW-1, Olympus) preloaded with $1 \mathrm{~mL}$ of indigo carmine was gently inserted through the working channel of the bronchoscope into each target bronchus and then injected 
under fluoroscopic confirmation. After awakening the patient, another CT scan was performed to localize the actual markings to adjust for possible dislocation from the original plan, as reported previously. ${ }^{20}$ The CT images were reconstructed into 3 dimensions. Because injected indigo carmine remains visible in the lung for up to 2 days, ${ }^{10}$ surgery was conducted on the same day as marking or 1 or 2 days after depending on the logistics (see Video 1 for the details of the technique). The patients were followed for 30 days after the planned day of surgery. When an adverse event was detected, the affected patients were followed for more than 30 days.

\section{End Points}

The primary end point was successful resection, which was defined as resection of the lesion with resection margins larger than or equal to the lesion diameter or $2 \mathrm{~cm}$ using the initial planned resection. The resection was considered unsuccessful if the resection margins measured intraoperatively were insufficient and additional resection was conducted. The resection was considered unsuccessful regardless of the resection margins if insufficient lung mapping or insufficient localization of the lesion necessitated changes in the operation plan (eg, conversion from wedge resection to segmentectomy) or approach (eg, conversion from video-assisted thoracic surgery to open thoracotomy for palpation). In patients in whom the success of the resection could not be simply judged on the basis of the electronic case reporting form, an objective judgment was made by the event evaluation committee composed of a third party.

Resection margins were measured macroscopically by the surgeon intraoperatively while the lung was deflated. The registered lesion diameter measured by preoperative CT was used for the calculation. If the lesion was not identified macroscopically, and microscopic or pathologic examination revealed a compatible lesion, the lesion was considered successfully resected and the margin was measured in the pathology department, taking the width of the stapler into consideration.

We defined the primary goal of the study as the verification of a success proportion of not less than $90 \%$ and the achievement of successful resection in $95 \%$ of cases (lower limit of confidence interval [CI], 90\%) based on our retrospective data analysis of resection margins at the University of Tokyo that compared VAL-MAP, CT-guided marking, and no marking. ${ }^{21}$ The details are described in the study protocol (Online Data Supplement). The number of lesions required was calculated as 210 with the 1-sided significance level set at $2.5 \%$ and statistical power set at approximately $75 \%$ by which the lower threshold of $95 \%$ CI based on binomial distribution was higher than $90 \%$. In a previous study, each patient had an average of 1.35 lesions ${ }^{10}$; thus, an enrollment of 160 patients was expected to yield 210 lesions for analysis in the present study.

Secondary end points were (1) effectiveness of lung marking, (2) effectiveness of VAL-MAP-assisted surgery, and (3) safety. To evaluate the effectiveness of lung marking, the number of markings identified intraoperatively and the number of conducted markings were calculated. Successful localization of the lesion was evaluated regarding the whole lung map (combination of multiple markings). The effectiveness of surgery was evaluated as the effectiveness of the surgical approach (thoracoscopy alone vs palpation via a small thoracotomy) and resection method (wedge resection alone vs enlarged resection). The contribution degree of the marking to surgery was evaluated by the surgeon. Operation time was recorded. In regard to safety, data were collected on adverse events that occurred during and after the bronchoscopic procedure, and before, during, and after surgery up to postoperative day 30 .

\section{Data Collection and Analysis}

All data were collected using electronic data recorded at each institution. All data analyses were conducted independently by specialized statisticians according to the study protocol. The data were expressed as mean \pm standard deviation and median (min-max) when appropriate. Success rate of resection was assessed, and the $95 \%$ CI was calculated using the exact method. Also, potential risk factors assumed to affect resection failure were assessed using multivariable risk ratio regression model, including required resection depth, planned operation, measurement of resection margin on regular basis in the center, surgeons' average experience in thoracic surgery, and lesion characteristics as explanatory variables. To examine the more detailed relationship between the required resection depth and the resection failure/success, restricted cubic spline model was used, adjusting for the potential risk factors described. All reported $P$ values were 2-tailed. Statistical analyses were performed using SAS9.4 software (SAS Institute Inc, Cary, NC).

\section{Monitoring and Audit}

On-site monitoring of the quality and consistency of data collection was conducted at each institution. Central monitoring of data collection was also conducted. An audit was conducted at 2 representative institutions.

\section{RESULTS \\ Patients}

The consort diagram is shown in Figure 1. In total, 213 lesions from 162 cases (including patients registered twice for bilateral lesions) were primarily registered. Two patients with 3 lesions were excluded from the study, because the resections in these patients were canceled because of other concurrent lesions found after registration in 1 patient and a socioeconomic reason in the other patient; thus, 210 lesions in 160 patients underwent secondary registration. One patient did not undergo bronchoscopic marking because the patient requested postponement of the operation. The remaining 159 cases (155 patients) underwent further evaluation. Of these 155 patients, surgery was canceled in 3 patients with 4 lesions. In 2 of the patients for whom surgery was canceled, 2 lesions ( 1 in each patient) were found to have shrunk on postmapping CT and were clinically diagnosed as inflammatory change rather than malignant tumors; 1 of these 2 patients had another lesion also targeted by VAL-MAP and underwent surgery for this lesion as planned. The other patient with 2 lesions for whom surgery was canceled had cold-like symptoms, which exacerbated after bronchoscopy; thus, surgery was canceled, and this patient was then re-registered and underwent VAL-MAP and surgery after recovery. The remaining 157 patients underwent surgery as planned. However, because of unexpected intraoperative findings in 2 patients (pleural dissemination and fragility of tissue necessitating conversion from segmentectomy to wedge resection), unplanned resection was performed, and they were excluded from further analysis. Thus, planned resection was eventually performed for 203 lesions in 155 patients who were included for further analysis. The patient demographics of the full analysis set are shown in Table 1. The characteristics of targeted lesions are shown in Table 2. Among the 209 lesions, $121(57.9 \%)$ were less than $1 \mathrm{~cm}$ in diameter, 109 $(52.2 \%)$ were more than $1 \mathrm{~cm}$ in depth, and $59(28.2 \%)$ were less than $1 \mathrm{~cm}$ in diameter and more than $1 \mathrm{~cm}$ in depth. 


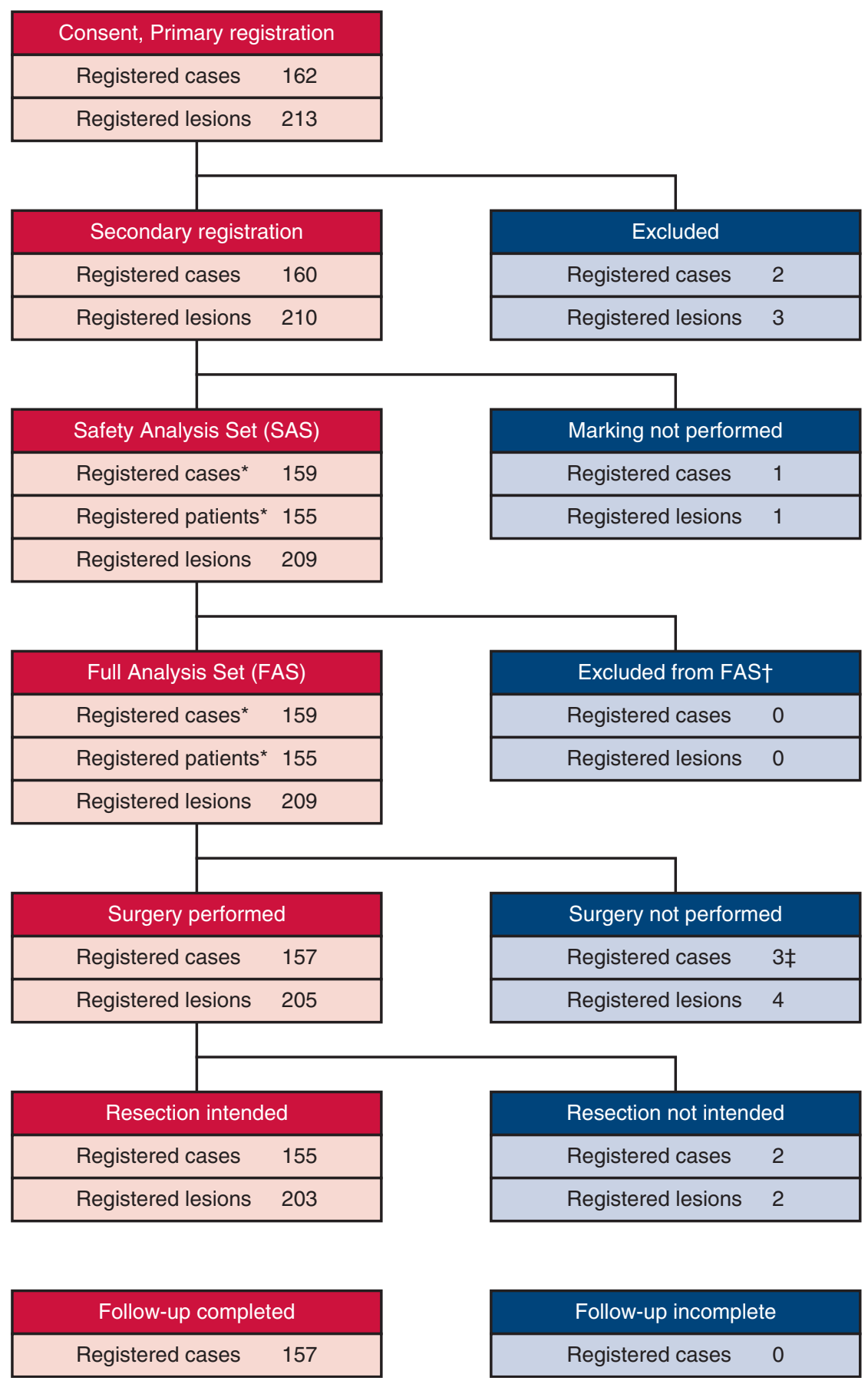

FIGURE 1. Consort diagram. *Some patients were registered twice, and these cases counted as 2 cases. $\nmid$ The reasons for exclusion from the FAS were deviation from the inclusion criteria, deviation from the exclusion criteria, no efficacy evaluation conducted after bronchoscopic marking, and other. $\ddagger$ In 1 patient, 1 lesion was not resected, and the other was resected.

\section{Primary End Point}

Among the 203 lesions in 153 patients who were eventually evaluated, 178 lesions met the criteria of successful resection $(87.7 \%$; 95\% CI, 82.4-91.9). Resection failure occurred in $12.3 \%$ (25/203 lesions). There were 131 patients in whom all lesions met the criteria for successful resection, and 136 patients had successful resection of at least 1 lesion. The most common reason for failure was insufficient surgical margins (19 lesions, $76 \%$ ), followed by changed operation plan due to insufficient lesion identification or insufficient marking (eg, change from wedge resection to segmentectomy: 12 lesions, 48\%), changed approach due to insufficient lesion identification or insufficient marking (eg, change 
TABLE 1. Patient demographics

\begin{tabular}{|c|c|c|c|}
\hline & Average & \multirow{2}{*}{\multicolumn{2}{|c|}{$\frac{\text { Median (min-max) }}{69(33-91)}$}} \\
\hline Age (y) & $68.1 \pm 10.1$ & & \\
\hline Height $(\mathrm{cm})$ & $160.37 \pm 9.33$ & \multicolumn{2}{|c|}{$160.0(135.3-180.0)$} \\
\hline Weight (kg) & $58.45 \pm 10.55$ & \multicolumn{2}{|c|}{$57.0(38.0-98.0)$} \\
\hline BMI & $22.661 \pm 3.199$ & \multicolumn{2}{|c|}{$22.2(14.3-33.9)$} \\
\hline Brinkman index & $453.66 \pm 701.0$ & \multicolumn{2}{|c|}{$500(0-500)$} \\
\hline & & Category & $\begin{array}{c}\text { No. of } \\
\text { cases }(\%)\end{array}$ \\
\hline Sex & & Male & $79(51.0)$ \\
\hline \multirow{5}{*}{\multicolumn{2}{|c|}{ Performance status }} & 0 & $141(91.0)$ \\
\hline & & 1 & $9(5.8)$ \\
\hline & & 2 & $2(1.3)$ \\
\hline & & 3 & $3(1.9)$ \\
\hline & & 4 & $0(0)$ \\
\hline \multicolumn{2}{|c|}{ History of primary lung cancer } & Yes & $13(8.4)$ \\
\hline \multicolumn{2}{|c|}{$\begin{array}{l}\text { History of malignancy other than } \\
\text { lung cancer }\end{array}$} & Yes & 77 (49.7) \\
\hline \multicolumn{2}{|l|}{ Smoking history } & Yes & $82(52.9)$ \\
\hline \multicolumn{2}{|c|}{$\begin{array}{l}\text { History of allergy associated with } \\
\text { drugs used in the procedure }\end{array}$} & Yes & $7(4.5)$ \\
\hline \multicolumn{2}{|c|}{ Present or past history of bronchial asthma } & Yes & $14(9.0)$ \\
\hline \multicolumn{2}{|c|}{ History of surgery on the same side } & Yes & $11(7.1)$ \\
\hline \multirow{3}{*}{\multicolumn{2}{|c|}{ Timing of surgery after VAL-MAP }} & Same day & $23(14.6)$ \\
\hline & & $1 \mathrm{~d}$ after & $119(75.8)$ \\
\hline & & $2 \mathrm{~d}$ after & $15(9.6)$ \\
\hline \multirow{4}{*}{\multicolumn{2}{|c|}{ No. of targeted lesions per case }} & 1 & $126(81.3)$ \\
\hline & & 2 & $16(10.3)$ \\
\hline & & 3 & $9(5.8)$ \\
\hline & & 4 & $4(2.6)$ \\
\hline
\end{tabular}

BMI, Body mass index; VAL-MAP, virtual-assisted lung mapping.

from video-assisted thoracic surgery to minithoracotomy: 2 lesions, $8 \%$ ), and resection not confirmed (4 lesions, 16\%). In some cases, resection failure was due to multiple reasons.

The success rate of resection varied among centers, ranging from $25 \%$ to $100 \%$ (Table E2). Among centers that registered more than 10 lesions, the success rate varied from $69.2 \%$ to $95.2 \%$. Success rate was also examined in different patient groups, according to smoking history, time from marking to operation, and lesion characteristics (Table 3). The result of multivariable analysis is shown in Table 4. The most significant factor leading to resection failure was the depth of the required resection line $(P=.0072)$. Measurement of resection margin performed occasionally or no routine measurement showed a higher risk compared with measurement on a regular basis in the center $(P=.044)$. Resection success was not associated with operation plan (wedge resection or segmentectomy), Brinkman index, or surgeons' experience. An ad hoc
TABLE 2. Characteristics of targeted lesions

\begin{tabular}{lrc}
\hline & Mean \pm SD & Median (min-max) \\
\hline Diameter $(\mathrm{mm})$ & $9.6 \pm 5.3$ & $8.0(2-34)$ \\
Depth $(\mathrm{mm})$ & $10.39 \pm 8.7$ & $10.0(0-45)$ \\
\hline Required resection depth $(\mathrm{mm})^{*}$ & $29.7 \pm 14.1$ & $28.0(6-71)$ \\
\hline \multicolumn{2}{c}{ Category } & No. of lesions (\%) \\
\hline CT characteristics & Pure GGO & $75(35.9)$ \\
& GGO with solid component & $36(17.2)$ \\
& Nodule/solid & $91(43.5)$ \\
& Cavity & $7(3.3)$ \\
Clinical diagnosis & Primary lung cancer $\dagger$ & $128(61.2)$ \\
& Metastatic lung tumor $\dagger$ & $80(38.3)$ \\
& Other & $1(0.5)$ \\
\hline
\end{tabular}

The number of lesions was 209, unless the number is indicated. $S D$, Standard deviation; $C T$, computed tomography; $G G O$, ground-glass opacity. *Required resection depth $(\mathrm{mm})=$ depth $($ distance from the closest pleura $)+\{$ [diameter $\times 2$ (tumor $<2 \mathrm{~cm}$ )] or [diameter +20 (tumor $\geq 2 \mathrm{~cm}$ )] $\}$. †ncluding suspected diagnosis.

analysis of the study demonstrated that the risk of resection failure (ie, insufficient resection margins) increased according to the depth of the resection line, reaching $10 \%$ when the resection line was deeper than $30 \mathrm{~mm}$ from the lung surface (Figure 2).

\section{Secondary End Point: Effectiveness of Marking}

Among 559 marks made, 521 marks were successfully identified intraoperatively $(93.2 \% ; 95 \%$ CI, 90.8-95.1). The grading (intensity) of the marking is shown in

TABLE 3. Resection success among different patient groups

\begin{tabular}{|c|c|c|c|c|}
\hline & $\begin{array}{c}\text { No. of } \\
\text { patients }\end{array}$ & $\begin{array}{c}\text { No. of } \\
\text { targeted } \\
\text { lesions }\end{array}$ & $\begin{array}{c}\text { Successfully } \\
\text { lesion } \\
\text { resected }(\%)\end{array}$ & $95 \%$ CI \\
\hline \multicolumn{5}{|l|}{ Smoking history } \\
\hline Yes & 71 & 97 & $87(89.7)$ & 81.9-94.9 \\
\hline No & 81 & 106 & $91(85.8)$ & 77.7-91.9 \\
\hline \multicolumn{5}{|l|}{$\begin{array}{l}\text { Timing of operation } \\
\text { after mapping* }\end{array}$} \\
\hline Same day & 23 & 36 & 33 (91.7) & $77.5-98.2$ \\
\hline $1 \mathrm{~d}$ after & 117 & 152 & $131(86.2)$ & 79.7-91.2 \\
\hline $2 \mathrm{~d}$ after & 15 & 15 & $14(93.3)$ & $68.1-99.8$ \\
\hline \multicolumn{5}{|l|}{ Brinkman Index } \\
\hline$<500$ & 96 & 129 & $113(87.6)$ & $80.6-92.7$ \\
\hline$\geq 500$ & 56 & 74 & $65(87.8)$ & $78.2-94.3$ \\
\hline \multicolumn{5}{|l|}{ Depth of lesion } \\
\hline$<2 \mathrm{~cm}$ & - & 174 & $155(89.1)$ & $83.5-93.3$ \\
\hline$\geq 2 \mathrm{~cm}$ & - & 29 & $23(79.3)$ & $60.3-92.0$ \\
\hline \multicolumn{5}{|c|}{ Required resection depth* } \\
\hline Less than median & - & 99 & $95(96.0)$ & $90.0-98.9$ \\
\hline $\begin{array}{l}\text { Equal to or greater } \\
\text { than median }\end{array}$ & - & 104 & $83(79.8)$ & $70.8-87.0$ \\
\hline
\end{tabular}

CI, Confidence interval. *Required resection depth $(\mathrm{mm})=$ depth (distance from the closest pleura) $+\{[$ diameter $\times 2$ (tumor $<2 \mathrm{~cm})]$ or $[$ diameter $+20($ tumor $\geq 2 \mathrm{~cm})]\}$. 
Table E3. Most marks (79.8\%) were graded as appropriate ( $\mathrm{G} 2$ and G3). The most common reason for a grading of G0 (invisible) or G1 (faint) was central injection, which caused technical marking failure in $30.8 \%$ (Table E4). A previous study suggested that a Brinkman index of 500 or greater is associated with invisibility of markings ${ }^{22}$; however, this relationship was not seen in the present study (marking success: Brinkman index $<500,92.8 \%$; Brinkman index $\geq 500,93.8 \%$ ). VAL-MAP markings successfully aided in the identification of 190 lesions $(93.6 \% ; 95 \%$ CI, 89.3-96.5). Resection of the lesion (regardless of additional resection or changes in surgical plan) was achieved in 199 lesions $(98.0 \%$; 95\% CI, 95.0-99.5).

\section{Secondary End Point: Effectiveness of Marking Assisted Surgery}

Regarding surgical approach, completely thoracoscopic surgery was performed in $86.0 \%$ of cases, followed by minithoracotomy with the aid of thoracoscopy $(11.5 \%)$ and open thoracotomy $(2.5 \%)$. The data on planned and actual approach are summarized in Table E5. Regarding changes in surgical approach, conversion from completely thoracoscopic surgery to minithoracotomy or open thoracotomy was needed in $1.4 \%$ and $0.7 \%$ of cases, respectively, and minithoracotomy was converted to open thoracotomy in $5.9 \%$ of cases. Otherwise, the planned approach was accomplished. Regarding resection method, there were 184 resections conducted, most commonly via wedge resection $(63.6 \%)$, segmentectomy $(27.2 \%)$, and wedge resection followed by lobectomy $(4.3 \%)$. The details and success ratio depending on resection type are shown in Table E6. There was no obvious difference in the incidence of successful resection between wedge resection and segmentectomy.

In patients in whom only 1 resection was conducted, the average times for wedge resection and segmentectomy were $93.2 \pm 57.6$ minutes and $202.4 \pm 63.2$ minutes, respectively. The contribution of VAL-MAP to surgery as evaluated by each surgeon per case was most commonly graded as "impossible to achieve accurate resection without mapping" in 85 cases $(54.1 \%)$, followed by "possible to achieve the same operation but mapping enabled confident resection" in 70 cases $(44.6 \%)$. Details of the perceived contribution of VAL-MAP to surgery are shown in Table E7.

\section{Secondary End Point: Safety}

Safety was evaluated in 155 patients who underwent bronchoscopic marking. During the study period from mapping to postoperative day 30 , adverse events regardless of the association with VAL-MAP were observed in 50 patients $(32.3 \%)$. The number of adverse events was $12(7.7 \%)$ during the bronchoscopic procedure, 32 (20.6\%) during postmapping CT, $5(3.2 \%)$ in the period from mapping to surgery, and $13(8.4 \%)$ postoperatively. The only major adverse event reported was postoperative delayed pleural fistula that necessitated readmission of the patient, although this event was judged as not associated with the VAL-MAP procedure. No patient death was reported. All reported adverse events are listed in Table E8.

Adverse events reported in more than $1 \%$ of cases were as follows. Events observed during bronchoscopy: hypertension $(3.2 \%)$, arrhythmia $(1.3 \%)$, and airway bleeding $(1.3 \%)$. Events observed during postmapping CT: bulla formation in the lung $(10.3 \%)$, pneumothorax $(10.3 \% ; 1$ patient $(0.6 \%)$ needed chest tube drainage), and mediastinal emphysema $(2.6 \%)$. Events observed during the period from mapping to surgery: fever higher than $38^{\circ} \mathrm{C}(1.9 \%)$. Events observed during the postoperative period: pleural fistula $(1.9 \%)$ and others $(3.9 \%)$. No major adverse event was directly associated with VAL-MAP.

Past or present history of bronchial asthma was seen in 14 patients $(9.6 \%)$. One patient developed a cough that necessitated interruption of the procedure during bronchoscopy, and 1 patient experienced an asthma attack after surgery. Otherwise, there was no trend of adverse events in patients with versus without asthma.

\section{DISCUSSION}

Previous multicenter research has revealed that VAL-MAP is safe, whereas conventional CT-guided percutaneous needle-mediated marking results in potentially fatal air embolism in $1 \%$ to $2 \%{ }^{4-7}$ Therefore, it was not ethically acceptable to conduct the present study as a controlled study. Moreover, although a previous multicenter study demonstrated a high successful resection rate of targeted lesions $(99.0 \%){ }^{10}$ the study did not evaluate resection margins. The management of patients with positive resection margins remains an important controversial issue, ${ }^{23}$ and even if the resection margins are negative, an insufficient resection margin distance has been demonstrated to affect local recurrence and patient survival. $^{14,15,24}$ Therefore, we set the primary end point of the present study as the successful resection rate, which was defined by the achievement of sufficient resection margins.

We set the primary goal of the study as achieving successful resection in $95 \%$ of cases, with a lower CI of $90 \%$. However, the eventual successful resection rate was $87.5 \%$. We consider that this result does not necessarily demonstrate ineffectiveness of the present VALMAP technique. Rather, we consider that it revealed an important limitation in applying the technique to a subgroup of targeted lesions and indicates a future research direction.

Multivariable analysis demonstrated that the depth of the resection line required to obtain a sufficient resection 
TABLE 4. Multivariable analysis of the risk factors affecting resection failure

\begin{tabular}{|c|c|c|c|c|}
\hline & Reference & Risk ratio & $95 \% \mathbf{C I}$ & $P$ value \\
\hline Required resection depth* equal to or greater than median & Less than median & 4.29 & $1.48-12.40$ & .0072 \\
\hline Planned operation: segmentectomy & Wedge resection & 0.73 & $0.34-1.55$ & .416 \\
\hline $\begin{array}{l}\text { Measurement of resection margin performed on a regular basis in the center: } \\
\text { occasional or no routine measurement }\end{array}$ & Regular measurement & 2.45 & $1.02-5.84$ & .044 \\
\hline Surgeons' average experience: per 5-y increase & & 1.05 & $0.63-1.76$ & .856 \\
\hline Lesion characteristics: GGO with solid component & Nodule/solid or cavity & 2.44 & $0.98-6.10$ & .055 \\
\hline Lesion characteristics: pure GGO & Nodule/solid or cavity & 1.56 & $0.62-3.91$ & .34 \\
\hline
\end{tabular}

$C I$, Confidence interval; $G G O$, ground-glass opacity. *Required resection depth $(\mathrm{mm})=$ depth (distance from the closest pleura) $+\{[$ diameter $\times 2$ (tumor $<2$ cm) $]$ or $[$ diameter $+20($ tumor $\geq 2 \mathrm{~cm})]\}$.

margin was the most significant factor affecting the success of resection (Table 4). Because of the nature of the lung marking placed on the lung surface, including VAL-MAP, this limitation had been theoretically predicted. ${ }^{8}$ Conversely, by making the resection large enough, particularly in wedge resection, this limitation was considered to be conquerable to some extent according to the surgeon's experience, thoughtful mapping design, and surgical strategy. ${ }^{8,11,12}$ Moreover, the application of segmentectomy is considered to eliminate the problem. The present study clarified that the deep resection margin needs even more careful determination, even in segmentectomy in which a fan-shaped resection results in shorter resection margins for a deeply located lesion. In particular, if the required depth of resection

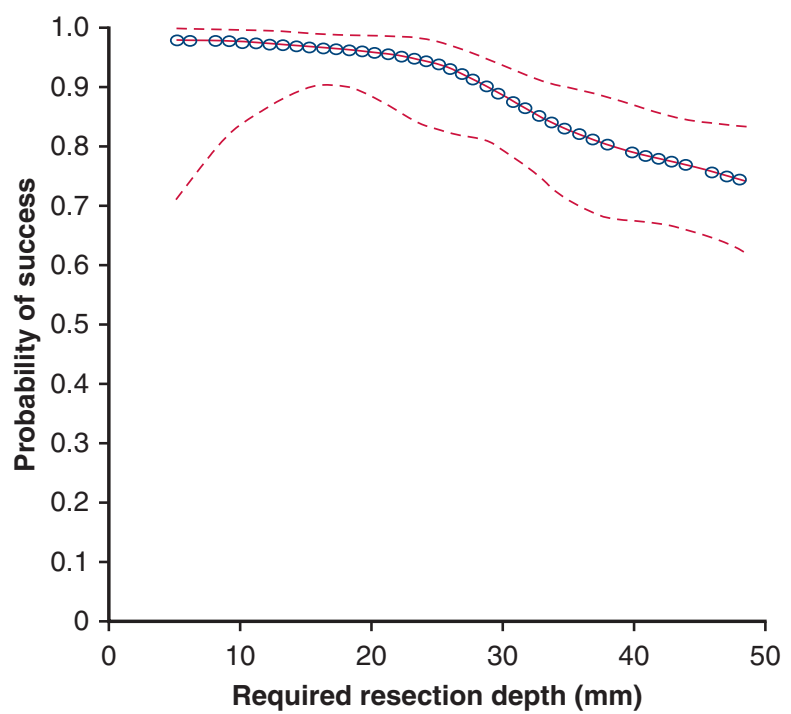

FIGURE 2. The relationship between required resection depth and risk of resection failure. A restricted cubic spine regression depicting the relationship between probability of resection success and required depth of resection line $(\mathrm{mm})$ calculated by depth (distance from the closest pleura) $+\{$ [diameter $\times 2$ (tumor $<2 \mathrm{~cm}$ )] or [diameter +20 (tumor $\geq 2 \mathrm{~cm}$ )] . The interrupted curves indicate $95 \% \mathrm{CI}$. was more than $30 \mathrm{~mm}$, the risk of resection failure was greater than $10 \%$ (Figure 2). Although the data are based on our single study and validation is yet to be done, this result indicates that such cases require special attention or reconsideration of the surgical plan. We are developing improvements in the VAL-MAP technique by not limiting it to the lung surface, but extending it to deeper regions (ie, 3-dimensional mapping). We are initiating a clinical trial combining the current VAL-MAP technique with bronchoscopic coil marking to indicate the deep resection line. The VAL-MAP technique is still being developed, and the present study played a critical role in revealing the current limitation and an important future direction.

It was challenging to set an appropriate goal in the present single-arm study. The goal of the present study was set on the basis of previous internal data of VAL-MAP in a single institution and histologic data of conventional CT-guided marking in the same institution, both of which could have been biased. Because data on resection margins were not collected in the previous multicenter study, ${ }^{10}$ we used data from an institution where the data on resection margins were routinely collected. Although the data showed successful resection in 47 of 49 lesions by the definition of success in the present study $(95.8 \%)$, we did not take center-to-center variation into consideration. Indeed, the outcome of the present study showed high variance among centers (Table E2). It is possible that the present results were biased by the large number of centers and operators involved, with a relatively low number of included patients. Moreover, among participating centers where the resection margins had been routinely measured, the successful resection rate reached $94.1 \%$, suggesting that this was a significant factor affecting successful resection (Table 4). Therefore, the hands-on practice used by surgeons to measure resection margins may be surprisingly important for successful resection, although the importance of resection margins has been generally recognized by most surgeons. 
The historical data of CT-guided marking also may have been biased. We estimated a maximum success rate of $85 \%$ in the historical control, assuming that all the cases with incomplete descriptions of resection margins were successful. Although the actual success rate among cases with full descriptions of resection margins was only $67 \%$, we considered the possibility that resection margins tended to be reported when the resection margin was insufficient. Once again, for ethical and safety reasons, a direct comparison between the 2 marking methods was avoided in the present study, which was a limitation of this prospective study. Because safety was a concern with conventional CT-guided marking and indeed motivated us to develop the VAL-MAP method, ${ }^{3,8}$ the present study reinforced the evidence on the safety of VAL-MAP as a secondary end point that was demonstrated in a previous multicenter study. ${ }^{10}$

Notably, the other secondary end points of effectiveness of marking, effectiveness of marking-assisted surgery, and safety demonstrated satisfactory outcomes. This suggests that the VAL-MAP technique was appropriately conducted in centers in most cases, whereas many cases of resection failure occurred despite the success of lung mapping. Therefore, together with the outcome of a previous study, ${ }^{10}$ the current VAL-MAP technique is considered to be well established and well reproducible in various centers. However, it is important for surgeons to pay careful attention to resection margins, even with VAL-MAP, and the current VAL-MAP technique needs further improvement when targeting a lesion that necessitates a deep resection line.

The present study did not investigate the long-term outcome of patients who underwent sublobar lung resection. Insufficient resection margins reportedly lead to frequent local recurrence ${ }^{13-16}$ and poorer clinical outcomes. ${ }^{17}$ The present study allowed for additional resection or changes in operation plan if surgeons thought that the resection margin was insufficient; taking this into consideration, an ultimately successful resection with satisfactory margins was achieved in $98.0 \%$. Evaluation of such long-term end points will reveal the eventual clinical usefulness of the technique. Notably, the current VAL-MAP technique necessitates an additional bronchoscopic procedure and CT scan, which adds an extra cost on top of the cost of surgery. Thus, a cost-benefit analysis of the use of VAL-MAP should be performed.

\section{CONCLUSIONS}

The present study demonstrated the reasonable efficacy and high safety of VAL-MAP. The depth of the required margin was the most significant factor leading to resection failure when using the present VAL-MAP technique. Further investigation is undergoing to overcome this challenge.

\section{Webcast}

You can watch a Webcast of this AATS meeting presentation by going to: https://aats.blob.core.windows. net/media/18Apr30/25ABC \%202.General\%20Thoracic\% 20SS/S69\%20-\%20Part\%201/S69_3_webcast_02400 1840.mp4.

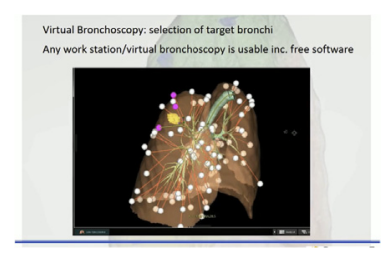

\section{Conflict of Interest Statement}

Authors have nothing to disclose with regard to commercial support.

The authors thank the staff from all the centers that participated in the present study, especially Dr Kazuhiro Nagayama (The University of Tokyo), Dr Kenichi Okubo (Tokyo Medical and Dental University), Drs Toru Bando and Atsushi Kitamura (St Luke's International Hospital, Tokyo), Dr Shinji Shinohara (University of Occupational and Environmental Health, Kitakyushyu), Dr Jun Matsumoto (NTT Medical Center Tokyo), Dr Taiki Hoshino (Shimane Prefectural Central Hospital, Izumo), Drs Yoshiaki Furuhata and Yuriko Terada (Japanese Red Cross Medical Center, Tokyo), Dr Kenji Misawa' (Aizawa Hospital, Matsumoto), Drs Takuji Fujinaga and Masaki Ikeda (Nagara Medical Center, Gifu), Drs Noritaka Isowa and Hideaki Miyamoto (Matsue Red Cross Hospital, Matsue), Drs Ryotaro Kamohara and Keitaro Matsumoto (Nagasaki University), Drs Yukihiro Yoshida and Takafusa Yoshioka (Asahi General Hospital), Drs Hiroaki Sakai and Yuki Morimura (Hyogo Prefectural Amagasaki General Medical Center), Dr Yasuo Sekine (Tokyo Women's Medical University Yachiyo Medical Center), Dr Terumoto Koike (Niigata University Graduate School of Medical and Dental Sciences), Dr Yosuke Otake (Kitano Hospital, Osaka), and Dr Kenji Suzuki (Juntendo University, Tokyo). The authors thank the members of the clinical research support center at the University of Tokyo Hospital, especially Tomohiro Haga (project officer), Dr Keiko Ueda (general manager), Drs Yukari Uemura and Takuya Kawahara (statistics), Drs Munenori Takata and Mariko Takeda (data management officers), Makoto Akabori, Yumi Tanaka, and Ai Okazaki (monitoring officers), Ikue Wada and Fumiko Watanabe (Safety), and Mari Hirano (general assistance). The authors thank Hisae Hase and Shiori Toyomaru (Medical Professions Division of the University of Tokyo Hospital) for assisting with the arrangement of the study. The authors thank the Clinical Research Governance Department, Audit and Reliability Assurance Room, the University of Tokyo, especially Yuki Kusaka, Yoshie Yamashita, and Naho Sakakibara for auditing. The authors thank Dr Yasuhiro Hida (Hokkaido University, Sapporo), Dr Yasushi Matsuda (Tohoku University, Sendai), and Dr Takekazu Iwata (Chiba Cancer Center) for participating in the study as external members of the Effect Safety Evaluation Committee, Event Evaluation Committee, or Adverse Event Analysis committee. The authors thank Dr Kelly Zammit, BVSc, from Edanz Group (www. edanzediting.com/ac), for editing a draft of this manuscript. 


\section{References}

1. Higashiyama M, Tokunaga T, Nakagiri T, Ishida D, Kuno H, Okami J. Pulmonary metastasectomy: outcomes and issues according to the type of surgical resection. Gen Thorac Cardiovasc Surg. 2015;63:320-30.

2. Iwata H. Therapeutic strategy for small-sized lung cancer. Gen Thorac Cardiovasc Surg. 2016;64:450-6.

3. Sato M, Omasa M, Chen F, Sato T, Sonobe M, Bando T, et al. Use of virtual assisted lung mapping (VAL-MAP), a bronchoscopic multispot dye-marking technique using virtual images, for precise navigation of thoracoscopic sublobar lung resection. J Thorac Cardiovasc Surg. 2014;147:1813-9.

4. Iguchi T, Yoshioka T, Muro M, Miyasho K, Inoue D, Hiraki T, et al. Systemic air embolism during preoperative pulmonary marking with a short hook wire and suture system under CT fluoroscopy guidance. Jpn J Radiol. 2009;27:385-8.

5. Sakiyama S, Kondo K, Matsuoka H, Yoshida M, Miyoshi T, Yoshida S, et al. Fatal air embolism during computed tomography-guided pulmonary marking with a hook-type marker. J Thorac Cardiovasc Surg. 2003;126:1207-9.

6. Horan TA, Pinheiro PM, Araujo LM, Santiago FF, Rodrigues MR. Massive gas embolism during pulmonary nodule hook wire localization. Ann Thorac Surg. 2002; 73:1647-9.

7. Kamiyoshihara M, Sakata K, Ishikawa S, Morishita Y. Cerebral arterial air embolism following CT-guided lung needle marking. Report of a case. J Cardiovasc Surg (Torino). 2001;42:699-700.

8. Sato M. Virtual assisted lung mapping: navigational thoracoscopic lung resection. Cancer Res Front. 2016;2:85-104.

9. Sato M, Yamada T, Menju T, Aoyama A, Sato T, Chen F, et al. Virtual-assisted lung mapping: outcome of 100 consecutive cases in a single institute. Eur J Cardiothorac Surg. 2015;47:e131-9.

10. Sato M, Kuwata T, Yamanashi K, Kitamura A, Misawa K, Imashimizu K, et al. Safety and reproducibility of virtual-assisted lung mapping: a multicentre study in Japan. Eur J Cardiothorac Surg. 2017;51:861-8.

11. Sato M, Murayama T, Nakajima J. Techniques of stapler-based navigational thoracoscopic segmentectomy using virtual assisted lung mapping (VAL-MAP). J Thorac Dis. 2016;8:S716-30.

12. Sato M, Aoyama A, Yamada T, Menjyu T, Chen F, Sato T, et al. Thoracoscopic wedge lung resection using virtual-assisted lung mapping. Asian Cardiovasc Thorac Ann. 2015;23:46-54.

13. Schuchert MJ, Pettiford BL, Keeley S, D'Amato TA, Kilic A, Close J, et al. Anatomic segmentectomy in the treatment of stage I non-small cell lung cancer. Ann Thorac Surg. 2007;84:926-33.

14. Sawabata N, Ohta M, Matsumura A, Nakagawa K, Hirano H, Maeda H, et al. Optimal distance of malignant negative margin in excision of nonsmall cell lung cancer: a multicenter prospective study. Ann Thorac Surg. 2004;77:415-20.

15. Goldstein NS, Ferkowicz M, Kestin L, Chmielewski GW, Welsh RJ. Wedge resection margin distances and residual adenocarcinoma in lobectomy specimens. Am J Clin Pathol. 2003;120:720-4.

16. El-Sherif A, Fernando HC, Santos R, Pettiford B, Luketich JD, Close JM, et al. Margin and local recurrence after sublobar resection of non-small cell lung cancer. Ann Surg Oncol. 2007; 14:2400-5.

17. Wolf AS, Swanson SJ, Yip R, Liu B, Tarras ES, Yankelevitz DF, et al. The Impact of margins on outcomes after wedge resection for stage I non-small cell lung cancer. Ann Thorac Surg. 2017;4:1171-8.

18. The Japan Society for Respiratory Endoscopy. The Bronchoscope Manual [in Japanese]. 3rd ed. Available at: http://www.jsre.org/medical/anzen_tebiki_3. pdf. Accessed August 7, 2018.

19. Du Rand IA, Blaikley J, Booton R, et al. British Thoracic Society guideline for diagnostic flexible bronchoscopy in adults: accredited by NICE. Thorax. 2013; 68(Suppl 1):i1-44.

20. Sato M, Nagayama K, Kuwano H, Nitadori JI, Anraku M, Nakajima J. Role of post-mapping computed tomography in virtual-assisted lung mapping. Asian Cardiovasc Thorac Ann. 2017;25:123-30.

21. Kawashima S, Nagayama K, Sato M, Shinohara Y, Yoshida D, Terada Y, et al. Comparative review of sublobar resection before and after virtual assisted lung mapping introduction in a single institution. Interact Cardiovasc Thorac Surg. 2016;23:i21.

22. Yamanashi K, Sato M, Marumo S, Fukui T, Sumitomo R, Shoji T, et al. Emphysematous lungs do not affect visibility of virtual-assisted lung mapping. Asian Cardiovasc Thorac Ann. 2016;24:152-7.

23. Smeltzer MP, Lin CC, Kong FS, Jemal A, Osarogiagbon RU. Survival impact of postoperative therapy modalities according to margin status in non-small cell lung cancer patients in the United States. J Thorac Cardiovasc Surg. 2017;154:661-72.
24. Sakurai H, Asamura H. Sublobar resection for early-stage lung cancer. Transt Lung Cancer Res. 2014;3:164-72.

Key Words: ground glass opacity, lung cancer, metastatic lung tumor, wedge resection, segmentectomy

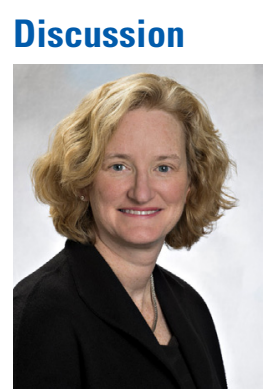

Dr Y. Colson (Boston, Mass). The only disclosure that $I$ have is an equipment loan for a camera from Novadaq, but otherwise, none.

As you have highlighted, this intraoperative localization of these small pulmonary nodules is an important challenge, and I know in the United States with lung cancer screening we are thinking it's approximately 1.5 to 2 million people. I assume it's similar in Japan in terms of incidence. As you have highlighted, those that are nonpalpable or located deep are challenging, and you have shown that in your study.

There are a variety of different ways people are talking about actually localizing these nodules. Some are actually using physical markers, some use dye, some are using molecular markers, and others are doing more adjunct imaging modalities. They all have risks and benefits, and I think you have shown that yours is safe and low risk, and has minimal potential for side effects. However, the ideal localization has to be both technically and logistically feasible and add value to the care of the patients. Given this, there are several questions.

Right upper lobe lesions, for example, can be technically challenging to reach by a navigational bronchoscopy approach to put your dye in. Is that a challenge for you on the VAL-MAP in certain locations, especially to get multiple areas of marking? If so, how many of the patients had tumors in those locations? Were you able to reach them or were they excluded because they weren't considered to be appropriate for the study?

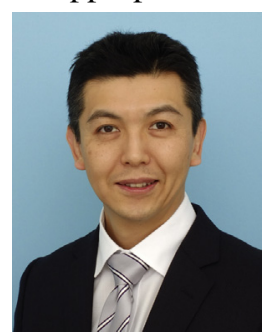

Dr Masaaki Sato (Tokyo, Japan). Sorry, I didn't quite understand the question.

Dr Colson. The difficulty in reaching specific lesions, for example, the right upper lobe can be hard to get the catheter all the way up and administer dye in multiple locations, or do you not have any challenging locations for tumors with VAL-MAP?

Dr Sato. In terms of the locations, basically we do not have any challenge. You can even put the dye on, for example, the diaphragmatic side or mediastinal side. Of course, the number 
of bronchi may be limited. The good thing about this mapping technique is you can put multiple marks that are basically a reference. So it does not really have to go to the target lesion itself. If you have a reference relatively close to the tumor, then you can calculate how much distance you have from the tumor, or something like that.

Dr Colson. So just by looking at your preoperative CT you can figure out which of the different bronchus you are going to get to?

Dr Sato. Exactly. And you can adjust the eventual mark with a postmarking CT.

Dr Colson. In terms of logistic feasibility, VAL-MAP requires the patient to go for preoperatively marking as a separate procedure, then a postmapping CT scan, and then the operating room, which is logistically challenging and can involve multiple days. Is there some shift in terms of your CT scan? When you look at the postinjection CT in the operating room, how much value is that really adding, that repeat second CT?

Dr Sato. We did a study to look at how much difference we have in the original plan and postmapping CT, and, on average, there is a $2.5-\mathrm{cm}$ difference. It was a little surprising to us. Still, there is no problem in doing the operation, because postmapping CT and the reconstructed 3-dimensional image show exactly the same as what we are supposed to see during the operation.

In terms of logistics, that's a very important point. At the beginning, I was concerned about logistics. Of course, in different parts of the world different logistics exist. In Japan, actually it works pretty well. Although we need another CT scan after mapping, it does not prolong the operation time at all. So we can do it completely outside of the operation room and finish the preparation before the day of operation. So we are absolutely relaxed at the time of operation.

On the other hand, maybe you want to do the mapping and operation all at once. That is something we are now trying using ENMB, and using ENMB we can actually do basically the same thing, and probably electromagnetic navigation bronchoscopy can eliminate the second CT scan process. That is what we are trying now. Hopefully we can present the data next year or so.

Dr Colson. Last, with all these different markings you can see on the surface, it is really about the depth, as you have highlighted. When you had several days in between your marking, does the dye spread on the surface making it hard to know where to remove, and have you looked for dye at the margin to see if that gives you an idea of how deep you need to go?

Dr Sato. After a couple of days it diffuses a little bit, but you can easily identify the center of the original mark, and it is not really a problem. However, in terms of the depth, it does not help so much in our experience. So to overcome the depth problem, we are starting a new clinical trial, basically placing a kind of fiducial marker at the time of dye marking on the bronchoscope, and it is helping a lot. So that's another avenue we are trying to pursue.

Dr Colson. Do you have dye in the lymphatics and lymph nodes or not?

Dr Sato. It was not so clear.

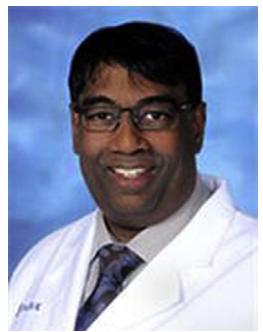

Dr H. Fernando (Falls Church, Va). That was a great presentation. You didn't meet your primary goal, and a part of it you alluded to was bringing this into the real world. So I wondered for the trial how you credentialed the different sites that you had and was there a minimum number that surgeons had to do before they could be part of the trial, and did you analyze for differences between sites for your success rate?

Dr Sato. Could you repeat that question again?

Dr Fernando. I am wondering about the variability between surgeons or sites and how you trained or credentialed to be part of the study and were there differences in the results between sites?

Dr Sato. People can learn the technique very quickly, so I was kind of relying on other people. Actually, that is not really the marking problem, as I said. Rather, it is the depth, so the angle of the stapling, that probably matters. It is a hard problem to solve.

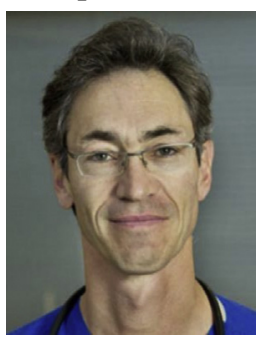

Dr J. Shrager (Stanford, Calif). It's clear in the literature, and in the Asian literature in particular, that a nodule, any on the adenocarcinoma spectrum that's less than $1 \mathrm{~cm}$ in size and less than $50 \%$ solid, is at essentially no risk of spreading, and if you let it grow a little bit, even, in fact, as it approaches $1 \mathrm{~cm}$, they are almost always palpable. So if you let it grow a bit, which is completely safe, then it becomes palpable and you have no issue finding and resecting it. Because of this, I am seeing few patients in whom I would find the expense and inconvenience of this technique and a lot of other marking procedures to be useful. So how would you counter that?

Dr Sato. Of course, there is a different way of thinking. I would say some patients, for example, who have a 7-mm ground-glass nodule, the patient was so anxious, and there are some ground-glass nodules showing gradual growth over time, even if it's a subcentimeter. So that's solely depending on how we observe the patient and a different way of thinking. We are not just cutting every GGO, but selecting patients, obviously. 


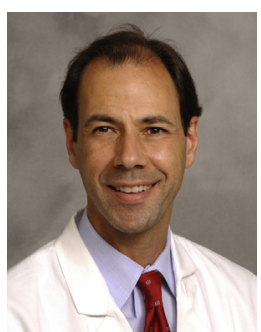

Dr T. D'Amico (Durham, NC). I have 2 questions. You said that $60 \%$ were wedge resections?

Dr Sato. Seventy percent

Dr D'Amico. And the rest were segmentectomies. How many of the failures were segmentectomies?

Dr Sato. The segmentectomy and wedge resection did not show any difference in terms of failure, a similar ratio of failure. It was surprising, because we thought segmentectomy would give us a better chance of a resection margin, but it was not really. So we also need to pay attention to the segmentectomy.

Dr D'Amico. What member of the operative team did the macroscopic measure of the margin?

Dr Sato. Usually the surgeons themselves.

Dr D'Amico. So it wasn't blinded in any way?

Dr Sato. No, it was not. 
TABLE E1. Inclusion and exclusion criteria. Patients meeting inclusion criteria I to III and not meeting the exclusion criteria completed primary registration after the provision of informed consent. Secondary registration was completed after approval by the case review committee and confirmation that each patient met all the inclusion criteria

I. A case in which pulmonary malignancy was suspected or diagnosed and the establishment of resection lines other than the typical interlobar fissure were required.

II. A case that required careful determination of resection lines to ensure resection margins because of difficult intraoperative tumor localization due to any of the following:

A) Lesions that were difficult to identify intraoperatively because of the characteristics of the lesion, including

i) lesions containing GGO in whole or in part;

ii) lesions with a tumor diameter of $\leq 5 \mathrm{~mm}$;

iii) lesions whose distance from the visceral pleura was larger than the tumor diameter.

B) Lesions that were difficult to identify intraoperatively because of underlying lung conditions:

i) Severe pleural adhesion was expected (eg, past history of open chest surgery);

ii) Preexisting benign nodules that were confusing and misleading (eg, silicosis, previous tuberculosis);

C) Lesions/conditions judged to require marking for other reasons (state the reasons for marking requirement).

III. The consent of the patient or substitute had been obtained.

IV. At the time of secondary registration, $\geq 2$ certified thoracic surgeons agreed that lung marking was necessary for resection.

Exclusion criteria:

I. Allergy to indigo carmine

II. Pregnancy

III. Bronchoscopy or marking could not be conducted because of existing complications.

IV. Other reasons that were considered by the corresponding or participating surgeons/physicians to render the patient inappropriate for inclusion in the study.

GGO, Ground-glass opacity.

TABLE E2. Successful resection rate among the participating centers

\begin{tabular}{lccc}
\hline Center & No. of targeted lesions & No. of successful resections (\%) & 95\% CI of successful resection \\
\hline A & 80 & $75(93.8)$ & $86.0-97.9$ \\
B & 27 & $24(88.9)$ & $70.8-97.6$ \\
C & 26 & $18(69.2)$ & $48.2-85.7$ \\
D & 21 & $20(95.2)$ & $76.2-99.9$ \\
E & 11 & $10(90.9)$ & $58.7-99.8$ \\
F & 11 & $10(90.9)$ & $58.7-99.8$ \\
G & 8 & $6(75.0)$ & $34.9-96.8$ \\
H & 4 & $1(25.0)$ & $0.6-80.6$ \\
\hline I & 3 & $3(100.0)$ & $29.2-100.0$ \\
J & 3 & $3(100.0)$ & $29.2-100.0$ \\
K & 2 & $1(50.0)$ & $1.3-98.7$ \\
\hline L & 2 & $2(100.0)$ & $15.8-100.0$ \\
M & 1 & $1(100.0)$ & $2.5-100.0$ \\
N & 1 & $1(100.0)$ & $2.5-100.0$ \\
O & 1 & $1(100.0)$ & $2.5-100.0$ \\
P & 1 & $1(100.0)$ & $2.5-100.0$ \\
\hline Q & 1 & $1(100.0)$ & $2.5-100.0$ \\
\hline
\end{tabular}

CI, Confidence interval 
TABLE E3. Graded marking intensity

\begin{tabular}{lcr}
\hline & No. of marks & $(\%)$ \\
\hline G0: invisible & 38 & 6.8 \\
G1: faint & 40 & 7.2 \\
G2: well visible, bleeding spot (-) & 317 & 56.7 \\
G3: well visible, bleeding spot (+) & 129 & 23.1 \\
G4: target-like marking & 16 & 2.9 \\
G5: bulla formation & 19 & 3.4 \\
\hline
\end{tabular}

TABLE E4. Reasons for invisible (G0) or faint (G1) marking

\begin{tabular}{lcr}
\hline & No. of marks & $(\%)$ \\
\hline Central injection & 24 & 30.8 \\
Severe anthracosis & 15 & 19.2 \\
Emphysematous lung & 12 & 15.4 \\
Thick pleura & 7 & 9.0 \\
Time lapse & 0 & 0.0 \\
Insufficient injection force & 11 & 14.1 \\
Did not reach the pleura because of an & 7 & 9.0 \\
$\quad$ intrapulmonary septum or other reason & & \\
Overlap of multiple marks & 6 & 7.7 \\
Unknown & 9 & 11.5 \\
Other & 5 & 6.4 \\
\hline
\end{tabular}


TABLE E5. Planned and actual surgical approach

\begin{tabular}{|c|c|c|c|c|c|c|}
\hline & \multicolumn{6}{|c|}{ Actual } \\
\hline & \multicolumn{2}{|c|}{ Complete VATS } & \multicolumn{2}{|c|}{$\begin{array}{c}\text { Minithoracotomy } \\
\text { (thoracoscopy assisted) }\end{array}$} & \multicolumn{2}{|c|}{ Open thoracotomy } \\
\hline & No. of cases (\%) & $95 \% \mathrm{CI}$ & No. of cases $(\%)$ & $95 \% \mathrm{CI}$ & No. of cases (\%) & $95 \% \mathrm{CI}$ \\
\hline \multicolumn{7}{|l|}{ Planned } \\
\hline Complete VATS & $135(97.8)$ & $93.8-99.5$ & $2(1.4)$ & $0.2-5.1$ & $1(0.7)$ & $0-4.0$ \\
\hline Minithoracotomy (thoracoscopy assisted) & $0(0)$ & $0-19.5$ & $16(94.1)$ & 71.3-99.9 & $1(5.9)$ & $0.1-28.7$ \\
\hline Open Thoracotomy & $0(0)$ & $0-84.2$ & $0(0)$ & $0-84.2$ & $2(100)$ & $15.8-100$ \\
\hline
\end{tabular}

VATS, Video-assisted thoracic surgery; $C I$, confidence interval.

TABLE E6. Planned and actual resection type

\begin{tabular}{|c|c|c|c|c|c|c|}
\hline \multirow[b]{2}{*}{ Resection type } & \multicolumn{6}{|c|}{ Actual } \\
\hline & $\begin{array}{l}\text { Wedge } \\
\text { resection }\end{array}$ & Segmentectomy & $\begin{array}{l}\text { Wedge resection } \\
\text { to lobectomy }\end{array}$ & $\begin{array}{l}\text { Wedge resection } \\
\text { to segmentectomy }\end{array}$ & $\begin{array}{l}\text { Segmentectomy } \\
\text { to lobectomy }\end{array}$ & Others* \\
\hline \multicolumn{7}{|l|}{ Planned } \\
\hline Wedge resection & $114(98.3)$ & $0(0)$ & $0(0)$ & $0(0)$ & $0(0)$ & $2(1.7)$ \\
\hline Segmentectomy & $2(3.8)$ & $50(96.2)$ & $0(0)$ & $0(0)$ & $0(0)$ & $0(0)$ \\
\hline Wedge resection to lobectomy & $1(10.0)$ & $0(0)$ & $8(80.0)$ & $1(10.0)$ & $0(0)$ & $0(0)$ \\
\hline Wedge resection to segmentectomy & $0(0)$ & $0(0)$ & $0(0)$ & $5(100.0)$ & $0(0)$ & $0(0)$ \\
\hline Segmentectomy to lobectomy & $0(0)$ & $0(0)$ & $0(0)$ & $0(0)$ & $1(100.0)$ & $0(0)$ \\
\hline Others & $0(0)$ & $0(0)$ & $0(0)$ & $0(0)$ & $0(0)$ & $0(0)$ \\
\hline
\end{tabular}

*Others indicates cases in which there were unexpected unusual changes in the surgical plan. In 1 patient, unexpected dissemination was found intraoperatively, and a wedge resection of the suspected dissemination was conducted instead of the planned operation. In the other patient, the markings were not clearly visible, and the planned wedge resection was not conducted (counted as a failure). 


\begin{tabular}{|c|c|c|}
\hline & Case & $(\%)$ \\
\hline \multicolumn{3}{|l|}{ Overall $(\mathrm{N}=127)$} \\
\hline Impossible to achieve accurate resection without mapping & 85 & 54.1 \\
\hline Possible to achieve the same operation, but mapping enabled confident resection & 70 & 44.6 \\
\hline Mapping was not necessary to achieve the same accuracy of operation & 2 & 1.3 \\
\hline \multicolumn{3}{|l|}{$\mathrm{CT}$ characteristics of the lesion } \\
\hline \multicolumn{3}{|l|}{ Pure GGO $(\mathrm{N}=46)$} \\
\hline Impossible to achieve accurate resection without mapping & 30 & 65.2 \\
\hline Possible to achieve the same operation, but mapping enabled confident resection & 16 & 34.8 \\
\hline Mapping was not necessary to achieve the same accuracy of operation & 0 & 0 \\
\hline \multicolumn{3}{|l|}{ GGO with solid component $(\mathrm{N}=35)$} \\
\hline Impossible to achieve accurate resection without mapping & 19 & 54.3 \\
\hline Possible to achieve the same operation but mapping enabled confident resection & 16 & 45.7 \\
\hline Mapping was not necessary to achieve the same accuracy of operation & 0 & 0 \\
\hline \multicolumn{3}{|l|}{ Nodule/solid $(\mathrm{N}=43)$} \\
\hline Impossible to achieve accurate resection without mapping & 12 & 27.9 \\
\hline Possible to achieve the same operation but mapping enabled confident resection & 29 & 67.4 \\
\hline Mapping was not necessary to achieve the same accuracy of operation & 2 & 4.7 \\
\hline \multicolumn{3}{|l|}{ Cavity $(\mathrm{N}=3)$} \\
\hline Impossible to achieve accurate resection without mapping & 2 & 66.7 \\
\hline Possible to achieve the same operation but mapping enabled confident resection & 1 & 33.3 \\
\hline Mapping was not necessary to achieve the same accuracy of operation & 0 & 0.0 \\
\hline \multicolumn{3}{|l|}{ Diameter of the lesion } \\
\hline \multicolumn{3}{|l|}{$<20 \mathrm{~mm}(\mathrm{~N}=113)$} \\
\hline Impossible to achieve accurate resection without mapping & 57 & 50.4 \\
\hline Possible to achieve the same operation but mapping enabled confident resection & 54 & 47.8 \\
\hline Mapping was not necessary to achieve the same accuracy of operation & 2 & 1.8 \\
\hline \multicolumn{3}{|l|}{$\geq 20 \mathrm{~mm}(\mathrm{~N}=14)$} \\
\hline Impossible to achieve accurate resection without mapping & 6 & 42.9 \\
\hline Possible to achieve the same operation but mapping enabled confident resection & 8 & 57.1 \\
\hline Mapping was not necessary to achieve the same accuracy of operation & 0 & 0.0 \\
\hline \multicolumn{3}{|l|}{ Depth of the lesion } \\
\hline \multicolumn{3}{|l|}{$<20 \mathrm{~mm}(\mathrm{~N}=110)$} \\
\hline Impossible to achieve accurate resection without mapping & 58 & 52.7 \\
\hline Possible to achieve the same operation, but mapping enabled confident resection & 50 & 45.5 \\
\hline Mapping was not necessary to achieve the same accuracy of operation & 2 & 1.8 \\
\hline \multicolumn{3}{|l|}{$\geq 20 \mathrm{~mm}(\mathrm{~N}=17)$} \\
\hline Impossible to achieve accurate resection without mapping & 5 & 29.4 \\
\hline Possible to achieve the same operation but mapping enabled confident resection & 12 & 70.6 \\
\hline Mapping was not necessary to achieve the same accuracy of operation & 0 & 0.0 \\
\hline
\end{tabular}

$C T$, Computed tomography; $G G O$, ground-glass opacity. 


\begin{tabular}{|c|c|c|c|}
\hline & No. of events & No. of cases & $(\%)$ \\
\hline \multicolumn{4}{|l|}{ During bronchoscopic procedure } \\
\hline Hypertension & 5 & 5 & 3.2 \\
\hline Hypotension & 0 & 0 & 0.0 \\
\hline Arrhythmia & 2 & 2 & 1.3 \\
\hline Hypoxia & 1 & 1 & 0.6 \\
\hline Wheeze & 0 & 0 & 0.0 \\
\hline Cough necessitating procedure interruption & 1 & 1 & 0.6 \\
\hline Restlessness & 1 & 1 & 0.6 \\
\hline Airway bleeding & 2 & 2 & 1.3 \\
\hline Pneumothorax & 0 & 0 & 0.0 \\
\hline Others & 0 & 0 & 0.0 \\
\hline Subtotal & 12 & 12 & 7.7 \\
\hline \multicolumn{4}{|l|}{ Confirmed at postmapping CT } \\
\hline Pneumothorax(no treatment needed) & 15 & 15 & 9.7 \\
\hline Pneumothorax (treatment needed) & 1 & 1 & 0.6 \\
\hline Bulla formation & 16 & 16 & 10.3 \\
\hline Mediastinal emphysema & 4 & 4 & 2.6 \\
\hline Alveolar bleeding & 1 & 1 & 0.6 \\
\hline Others & 0 & 0 & 0.0 \\
\hline Subtotal & 37 & 32 & 20.6 \\
\hline \multicolumn{4}{|l|}{ From mapping to operation } \\
\hline Prolonged conscious problem & 1 & 1 & 0.6 \\
\hline Paralysis & 0 & 0 & 0.0 \\
\hline Wheeze & 0 & 0 & 0.0 \\
\hline Hypoxia & 0 & 0 & 0.0 \\
\hline Fever $\left(\geq 38^{\circ} \mathrm{C}\right)$ & 3 & 3 & 1.9 \\
\hline Pneumonia & 0 & 0 & 0.0 \\
\hline Others & 1 & 1 & 0.6 \\
\hline Subtotal & 5 & 5 & 3.2 \\
\hline \multicolumn{4}{|l|}{ After surgery } \\
\hline Air leakage & 3 & 3 & 1.9 \\
\hline Interstitial pneumonia (exacerbation) & 0 & 0 & 0.0 \\
\hline Pneumonia & 1 & 1 & 0.6 \\
\hline Bronchial fistula & 0 & 0 & 0.0 \\
\hline Postoperative bleeding necessitating blood transfusion & 0 & 0 & 0.0 \\
\hline Respiratory failure & 0 & 0 & 0.0 \\
\hline Atelectasis (bronchoscopy needed) & 1 & 1 & 0.6 \\
\hline Pulmonary embolism & 0 & 0 & 0.0 \\
\hline Chylothorax & 0 & 0 & 0.0 \\
\hline Arrhythmia (treatment needed) & 1 & 1 & 0.6 \\
\hline Scar abscess & 1 & 1 & 0.6 \\
\hline Renal failure & 0 & 0 & 0.0 \\
\hline Empyema & 1 & 1 & 0.6 \\
\hline Mediastinitis & 0 & 0 & 0.0 \\
\hline Cerebral infarction & 0 & 0 & 0.0 \\
\hline Intracranial bleeding & 0 & 0 & 0.0 \\
\hline Myocardial infarction & 0 & 0 & 0.0 \\
\hline Reoperation within $24 \mathrm{~h}$ & 0 & 0 & 0.0 \\
\hline Others & 6 & 5 & 3.2 \\
\hline Subtotal & 14 & 13 & 8.4 \\
\hline
\end{tabular}

$C T$, Computed tomography. 